\title{
Relief of Chronic Posterior Neck Pain Depending on the Type of Forest Therapy: Comparison of the Therapeutic Effect of Forest Bathing Alone Versus Forest Bathing With Exercise
}

\author{
Boram Kang, MD ${ }^{1}$, Taikon Kim, MD, $\mathrm{PhD}^{1}$, Mi Jung Kim, MD, PhD ${ }^{1}$, Kyu Hoon Lee, MD, PhD ${ }^{1}$, \\ Seungyoung Choi, $\mathrm{MD}^{1}$, Dong Hun Lee, $\mathrm{MD}^{1}$, Hyo Ryoung Kim ${ }^{2}$, Byol Jun, $\mathrm{MA}^{2}$, \\ Seen Young Park ${ }^{2}$ Sung Jae Lee, MD, PhD ${ }^{2}$, Si-Bog Park, MD, PhD ${ }^{1}$
}

${ }^{1}$ Department of Rehabilitation Medicine, Hanyang University College of Medicine, Seoul;

Objective To compare the pain-reducing effect of forest bathing alone versus forest bathing in combination with stretching and strengthening exercises in patients with chronic posterior neck pain.

Methods Sixty-four subjects with posterior neck pain that had lasted more than 3 months were enrolled. They were randomly divided into a forest bathing alone (FBA) group and a forest bathing with exercise (FBE) group; each group included 32 subjects. All subjects from both groups walked every morning in the forest for about 2 hours for 5 days. In the afternoon, the FBE group did a stretching and strengthening exercise for about 4 hours; the FBA group had free time in the woods. Visual analog scale (VAS) on one day, VAS over the previous week, neck disability index (NDI), EuroQol 5D-3L VAS (EQ VAS) and index (EQ index), McGill pain questionnaire (MPQ), the number of trigger points in the posterior neck region (TRPs), and the range of motion of the cervical spine were evaluated on the first and last day of the program and compared between the two groups.

Results The number of TRPs were significantly reduced in the FBE group compared with the FBA group ( $\mathrm{p}=0.013)$. However, the other scales showed no significant difference between the two groups.

Conclusion When patients with chronic posterior neck pain underwent a short-term forest bathing (less than 7 days) program, FBE was more effective in the reduction of the number of TRPs than FBA. However, all other pain measurement scales we evaluated showed no statistically significant difference between the two protocols.

Keywords Neck pain, Exercise therapy, Chronic pain, Musculoskeletal pain, Complementary therapies

Received May 14, 2015; Accepted July 1, 2015

Corresponding author: Si-Bog Park

Department of Rehabilitation Medicine, Hanyang University Medical Center, Hanyang University College of Medicine, 222-1 Wangsimni-ro, Seongdong-gu, Seoul 04763, Korea

Tel: +82-2-2290-9226, Fax: +82-2-2290-9231, E-mail: sibopark@hanyang.ac.kr

(c) This is an open-access article distributed under the terms of the Creative Commons Attribution Non-Commercial License (http://creativecommons.org/ licenses/by-nc/4.0) which permits unrestricted noncommercial use, distribution, and reproduction in any medium, provided the original work is properly cited. Copyright () 2015 by Korean Academy of Rehabilitation Medicine 


\section{INTRODUCTION}

Posterior neck pain is a common complaint; it affects $70 \%$ of individuals at some time in their lives $[1,2]$. In many cases, it is difficult to pinpoint the cause of the pain. In cases where the underlying disease is not diagnosed and treated in the early stages, pain often does not respond well to general treatment and, as a result, the medical and socioeconomic costs of treatment can be very high. Indeed, the mechanism and role of chronic pain is different from that of acute pain. The major role of acute pain is the protection of the body from damage by noxious stimuli present in the external environment. On the contrary, chronic pain has more complex causes such as central nervous system damage, injury, or psychosocial problems, and it often has the characteristics of a pathological state rather than those of a physiological state [3]. Understanding of the importance of psychosocial factors such as affective and cognitive problems as a cause of chronic pain has gradually increased.

Although medication, surgical treatment, physical modalities, cervical traction, epidural injection, etc. have been applied for the purpose of treating posterior neck pain, there is seldom an optimal treatment, except in the case of surgical treatment for patients with specific conditions $[4,5]$. Because of the limitations of existing treatments, many complementary treatments have been suggested and researched. For instance, one study addressed pain reduction and increase in cervical range of motion (C-ROM) following dry needling and acupuncture in 36 patients with chronic neck pain [4]; another examined the pain-reducing effect of botulinum toxin A injection in 50 chronic neck pain patients [6]. However, no complementary treatments have been shown to be superior to existing medical interventions.

Recently, many studies on the therapeutic effects of the forest have been reported. A study in Japan showed an increase of natural killer cell activity after 3 days of forest bathing which lasted for a month [7]. A study comparing the physiological and psychological effects of forest versus urban environments on young male adults showed positive effects of forest bathing on physical and psychological health [8]. Several studies about the effects of forest bathing have been also reported in South Korea. Forest bathing was reported to have a positive impact on blood pressure and salivary cortisol level in elderly patients with hypertension [9] and therapeutic effects in patients with psychological problems such as anxiety, depression, and stress [10-12]. As far as we knew, however, a therapeutic effect of forest bathing on musculoskeletal pain had not yet been reported. Therefore, we previously conducted a preliminary study to address whether forest bathing may ameliorate chronic posterior neck pain and showed significantly reduced pain in a forest bathing group compared with a group going about daily life in a city. On the basis of this result, we hypothesized that forest bathing plus stretching and strengthening exercises targeted to the muscles in the cervical and shoulder regions would have a superior therapeutic effect compared with forest bathing alone; it has been shown that stretching and strengthening exercises are helpful for relieving posterior neck pain [13]. The therapeutic effect of exercise on neck pain is most likely because posterior neck pain may result in reflexive protective muscle spasms and decreased ROM, regardless of the primary cause of pain [14].

To test our hypothesis, we herein compare the pain-reducing effects of forest bathing alone versus forest bathing plus stretching and strengthening exercises targeted to the muscles of the cervical and shoulder regions.

\section{MATERIALS AND METHODS}

For one month in May 2013, 70 subjects who visited the Department of Rehabilitation Medicine of Hanyang University Medical Center and met certain inclusion criteria were recruited through a notice in the hospital, by phone, or by e-mail. Of these 70, 6 dropped out for personal reasons, so 64 subjects were finally included in this study. Subjects all gave voluntary informed consent prior to the beginning of the study.

Inclusion criteria were adults over the age of 20 with posterior neck pain graded more than 4 using the visual analog scale (VAS) that had lasted more than 3 months. Exclusion criteria were acute inflammation findings in blood tests, limitation of C-ROM because of cervical spinal fixation operations, inability to walk for more than an hour because of knee problems, or if residing in a forest for more than a week was a hardship for any reason including the presence of underlying disease.

Screening tests included simple radiographs of the cervical spine and both knee joints, routine blood tests, and 
VAS of cervical neck pain. On the first day of the experiment in the forest, 2 rehabilitation medicine physicians evaluated VAS on that day, VAS over the previous week, neck disability index (NDI), EuroQol 5D-3L VAS (EQ VAS) and index (EQ index), McGill pain questionnaire (MPQ), the number of trigger points in the posterior neck region (TRPs), and C-ROM. All the same tests were performed by the same physicians on the last day of the experiment.

The VAS was developed for evaluating factors that are difficult to objectively assess; it is commonly used for evaluating pain levels [15]. In general, patients are presented with a $10-\mathrm{cm}$ horizontal line anchored by 'no pain' on one end and 'pain as bad as it could be' on the other end; subjects mark the point on the line that they feel represents their pain level. The VAS score is determined by measuring length $(\mathrm{cm})$ on the line between the 'no pain' anchor and the subjects' mark. Authors defined 'VAS on one day' as the level of posterior neck pain on that day and 'VAS over the previous week' as an average pain level of the posterior neck region during the preceding week.

The NDI is a questionnaire developed in 1991 for the purpose of helping to understand how neck pain affects one's ability to manage everyday activities. It consists of 10 sections with 6 multiple choice questions per section [16].

The EQ VAS and index were developed by the EuroQol Group. They are standardized measurement tools that assess an individual's health status [17]. EQ VAS is evaluated in a similar manner as is VAS. Subjects mark their status on a vertical line anchored by 0 and 100 at either end to indicate 'Your own health state today.' A score of 0 means 'Worst imaginable health state' and a score of 100 means 'Best imaginable health state.' The EQ index has 5 sections, which are mobility, self-care, usual activities, pain/discomfort, and anxiety/depression, and each section has 3 possible answers.

The MPQ is a self-reported measure of pain level. It is composed of 78 words in 20 categories; for each category, subjects choose the word that best represents their feeling of pain. Scores are calculated by summing the values associated with each word and a higher score means more severe pain [18].

A TRP is defined as a hyperirritable spot and is diagnosed if a subject feels pain when weak pressure (less than $4 \mathrm{~kg} / \mathrm{cm}^{2}$ ) is applied with a pressure algometer [19]. In this study, the researchers examined bilateral cervical paraspinal, upper trapezius, and infraspinatus muscles, which are related to posterior neck pain, and counted the number of TRPs in these 6 muscles.

The C-ROM was evaluated by summating measures of cervical flexion, extension, right lateral bending, left lateral bending, right rotation, and left rotation.

Sixty-four subjects ( 11 males, 53 females) were randomly divided into a forest bathing alone (FBA) group $(\mathrm{n}=32)$ and a forest bathing with exercise (FBE) group $(\mathrm{n}=32)$. Both groups participated in a program consisting of 2 hours of walking in the forest every morning for 5 days. For 2 hours each afternoon, the FBE group did additional stretching and strengthening exercises targeting muscles in the cervical and shoulder regions. This exercise program was developed and organized by a committee composed of four physicians specializing in rehabilitation medicine and three physical therapists after a literature review. The exercise program consists of a 10-minute warm-up followed by 30 minutes of main

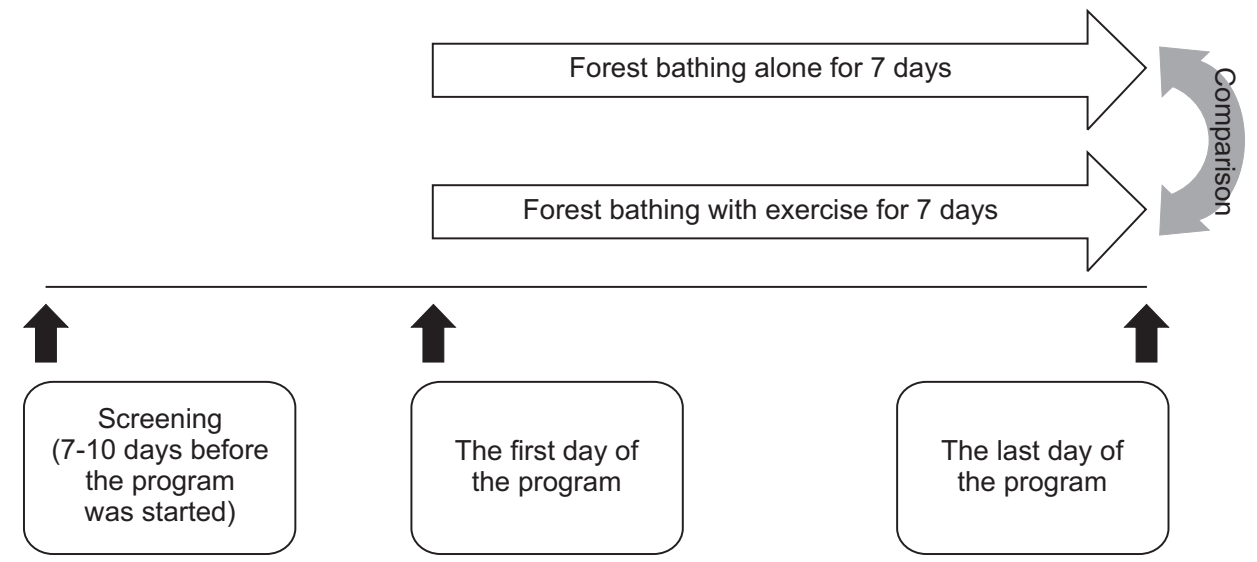

Fig. 1. Flow chart showing the schedule and plan of this study. 
exercise and a 10-minute cool down. Subjects rest for 10 minutes and then repeat the exercise program, so the total exercise time is 2 hours. The warm-up exercise is composed of light stretching; not only the cervical and shoulder regions but the whole body is included. In the main exercise, exercise intensity is gradually increased and stretching and strengthening exercises are focused on the cervical and shoulder regions. Although the cool down exercise is composed of only stretching, the intensity is higher than that of the main exercise. In contrast with the FBE group, the FBA group did not do the exercise program in the afternoon and instead had free time in the forest. After dinner, the FBE group did another 2 cycles of the same exercise program and the FBA group again had free time in the forest (Fig. 1).

Pain levels pre-intervention and post-intervention were compared between the FBA and FBE groups using the Mann-Whitney U test. The Wilcoxon signed-rank test was used to compare pre-intervention versus post-intervention outcomes in each group. The p-value for significance was set at 0.05 .

\section{RESULTS}

There was no significant difference in age, sex, height, or weight between the FBA and FBE groups. VAS on the day of screening and the first day of the forest program showed no significant difference between the two groups ( $\mathrm{p}=0.779$ and $\mathrm{p}=0.767$ ) (Table 1$)$. Most subjects were unemployed or students; only 5 subjects in the FBE group and 4 subjects in the FBA group had jobs. Of the employed subjects in the FBE group, 2 were office clerks, one

Table 1. Subject characteristics

\begin{tabular}{lccc}
\hline \multicolumn{1}{c}{ Variable } & Forest bathing alone $(\mathbf{n}=\mathbf{3 2})$ & Forest bathing with exercise $(\mathbf{n}=\mathbf{3 2})$ & p-value $^{\text {a) }}$ \\
\hline Age (yr) & $50.0 \pm 14.93$ & $54.8 \pm 9.78$ & 0.828 \\
\hline Sex (male:female) & $5: 27$ & $6: 26$ & 0.515 \\
Height $(\mathrm{cm})$ & $160.1 \pm 6.20$ & $160.7 \pm 6.81$ & 0.669 \\
Weight $(\mathrm{kg})$ & $58.4 \pm 10.17$ & $59.9 \pm 9.45$ & 0.985 \\
VAS at screening & $5.9 \pm 1.26$ & $6.4 \pm 1.33$ & 0.779 \\
\hline VAS on the first day & $5.2 \pm 1.61$ & $5.3 \pm 1.64$ & 0.767 \\
\hline
\end{tabular}

Values are presented as mean \pm standard deviation or number.

VAS, visual analog scale.

${ }^{a)} \mathrm{p}$-values were calculated using the independent $\mathrm{t}$-test ( $\mathrm{p}<0.05$, statistically significant).

Table 2. Difference between the two types of forest therapy program $(n=64)$

\begin{tabular}{|c|c|c|c|}
\hline & Forest bathing alone $(\mathrm{n}=32)$ & Forest bathing with exercise $(n=32)$ & p-value ${ }^{c)}$ \\
\hline VAS on one day ${ }^{\text {a) }}$ & $-2.7 \pm 1.69$ & $-2.9 \pm 1.93$ & 0.559 \\
\hline VAS over the previous week ${ }^{a}$ & $-2.8 \pm 1.40$ & $-3.1 \pm 1.77$ & 0.485 \\
\hline $\mathrm{NDI}^{\mathrm{a})}$ & $-15.1 \pm 8.45$ & $-11.3 \pm 11.73$ & 0.181 \\
\hline EQ VAS ${ }^{\mathrm{b})}$ & $28.7 \pm 15.12$ & $21.8 \pm 20.97$ & 0.165 \\
\hline EQ index ${ }^{b)}$ & $0.28 \pm 0.192$ & $0.22 \pm 0.154$ & 0.129 \\
\hline$M Q^{a)}$ & $-13.5 \pm 10.75$ & $-13.3 \pm 13.39$ & 0.652 \\
\hline $\mathrm{TRPs}^{\mathrm{a})}$ & $-1.0 \pm 1.49$ & $-2.1 \pm 1.66$ & $0.013^{*}$ \\
\hline C-ROM ${ }^{\text {b) }}$ & $74.7 \pm 60.69$ & $85.2 \pm 58.46$ & 0.667 \\
\hline
\end{tabular}

Values are presented as mean \pm standard deviation.

VAS, visual analog scale; NDI, neck disability index; EQ VAS, EuroQoL 5D-3L VAS; EQ index, EuroQol 5D-3L index; MPQ, McGill pain questionnaire; TRPs, trigger points in the posterior neck region; C-ROM, cervical range of motion.

${ }^{\text {a) }}$ Negative change indicates improvement.

${ }^{b)}$ Positive change indicates improvement.

${ }^{c} \mathrm{p}$-values were calculated using the Mann-Whitney U test.

${ }^{*} \mathrm{p}<0.05$, statistically significant. 
was self-employed, one worked as a translator, and one was a lecturer; in the FBA group, 2 were office clerks and 2 were self-employed.

Of all the pain assessment methods used to compare the two groups post-intervention, only the number of TRPs was significantly different; it was lower in the FBE group than in the FBA group ( $\mathrm{p}=0.013$ ). The other scales showed no significant differences between the two groups (Table 2).

All evaluated scales (VAS on a particular day, VAS over the previous week, NDI, EQ VAS and index, MPQ, the number of TRPs, and C-ROM) showed significant improvement after 7 days of the forest program in both groups (Table 3).

\section{DISCUSSION}

When we analyzed VAS on one day, VAS over the previous week, MPQ, and NDI, which reflect intensity of posterior neck pain, there were no significant differences between individuals in the two types of forest program. These results could be interpreted to mean that adding an exercise program to forest bathing is not an effective way to reduce posterior neck pain. EQ VAS and index, which reflect general health status, also showed no significant differences according to the type of forest program.

As mentioned above, posterior neck pain results in muscle spasms and decreased C-ROM in most cases regardless of the primary cause of pain. Therefore, we thought that evaluating the number of TRPs and C-ROM could be helpful for verifying pain intensity in the posterior neck region. C-ROM showed no significant difference between the two groups, like all other scales mentioned above. However, the number of TRPs was significantly reduced in the FBE group compared with the FBA group, which suggests that adding a stretching and strengthening exercise to forest bathing is more effective in reducing the number of TRPs than forest bathing alone.

It is unclear why intensity of pain, health status, and C-ROM were not significantly better in the FBE group in spite of a significant reduction in the number of TRPs. The authors' opinions about this issue are as follows. 1) The measurement method for detecting TRPs used in this study was a test for 'latent TRPs,' which are spots where pain is felt after applying weak pressure; these could be

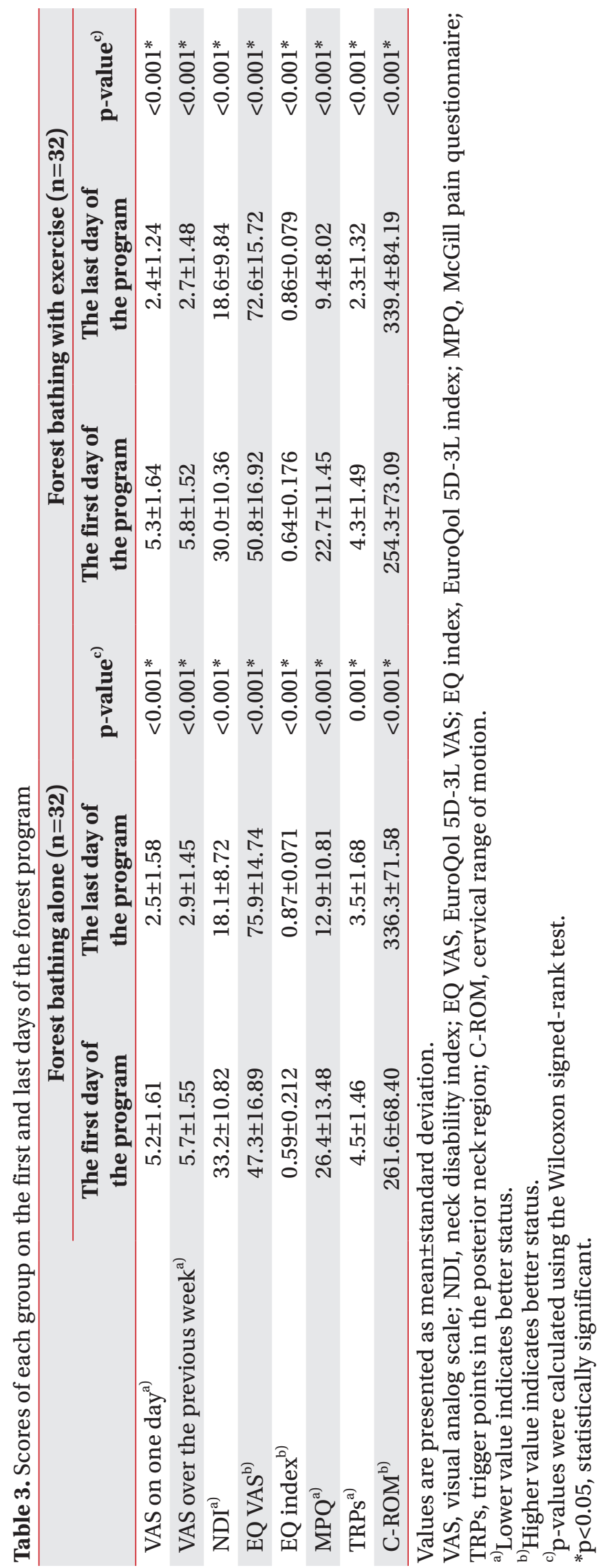


different from 'active TRPs,' which reflect pain subjects feel in the absence of any external pressure. Therefore, it is possible that, although the number of 'latent TRPs' was significantly reduced in the FBE group compared to the FBA group, the number of 'active TRPs' was not. 2) Because on average more than 2 TRPs still remained in patients in the FBE group, intensity of pain, health status, and C-ROM might not be affected. In addition, in this study, identifying the pain threshold of the remaining TRPs was impossible because a TRP was diagnosed if subjects felt pain less than $4 \mathrm{~kg} / \mathrm{cm}^{2}$ but other pressures were not tested. 3) TRPs do not necessarily reflect the main source of pain. Not only muscle but also bone, ligament, disc, nerve, etc. could be the focus of posterior neck pain. Likewise, C-ROM may remain limited because muscle pain is not the only cause of muscle spasms and limitations in ROM.

When comparing outcomes pre-intervention versus post-intervention, all scales showed significant improvement in both groups. This result suggests that forest bathing is effective in relieving pain and improving the health status of subjects with posterior neck pain. However, this conclusion has limitations because it is impossible to exclude the effect of simply resting on pain relief and health status improvement given the design of this study.

This study has several limitations. First, the duration of forest bathing was one week and the duration of the program was 5 days, which are short periods of time. Although the authors set the duration based on the duration of previous studies, most of which were 3 to 7 days, such short durations might be a cause of the insignificant effect of the stretching and exercise program. However, this short duration could be meaningful in respect to real life, in which many people spend from 2 or 3 days to about 7 days forest bathing during their weekends or vacations. In a follow-up study, we plan to compare the effect of the exercise program in longer-term forest bathing. Second, the ages of the subject did not show a normal distribution; specific ages were overrepresented. Because subjects had to make time to participate in a week-long study, students in their 20s and adults older than 50 made up the majority of the participants. As the effect of resting on pain relief and health status improvement could be limited to some degree because most of these subjects had no jobs, the result would be more meaningful if the next study includes patients with normally distributed ages and more patients who are employed. Third, as mentioned above, the effect of resting was not controlled. To solve this problem, in the next study, the FBA group must be compared with a group of participants who take a rest period in the city of the same duration.

In conclusion, when short-term forest bathing (less than 7 days) was used to treat patients with chronic posterior neck pain for the purposes of pain relief and health status improvement, FBE is more effective in the reduction of the number of TRPs than FBA. However, all other scales we evaluated showed no statistically significant difference between the two protocols.

\section{CONFLICT OF INTEREST}

No potential conflict of interest relevant to this article was reported.

\section{ACKNOWLEDGMENTS}

This research is supported by Ministry of Culture, Sports and Tourism (MCST) and Korea Creative Content Agency (KOCCA) in the Culture Technology (CT) Research \& Development Program 2015 and Korea Forest Service.

\section{REFERENCES}

1. Bronfort G, Evans R, Nelson B, Aker PD, Goldsmith $\mathrm{CH}$, Vernon $\mathrm{H}$. A randomized clinical trial of exercise and spinal manipulation for patients with chronic neck pain. Spine (Phila Pa 1976) 2001;26:788-99.

2. Cote P, Cassidy JD, Carroll L. The Saskatchewan health and back pain survey: the prevalence of neck pain and related disability in Saskatchewan adults. Spine (Phila Pa 1976) 1998;23:1689-98.

3. Braddom RL. Physical medicine and rehabilitation. 4th ed. Philadelphia: Saunders/Elsevier; 2011.

4. Irnich D, Behrens N, Gleditsch JM, Stor W, Schreiber MA, Schops P, et al. Immediate effects of dry needling and acupuncture at distant points in chronic neck pain: results of a randomized, double-blind, shamcontrolled crossover trial. Pain 2002;99:83-9.

5. Aker PD, Gross AR, Goldsmith CH, Peloso P. Conservative management of mechanical neck pain: systematic overview and meta-analysis. BMJ 1996;313:1291-6.

6. Wheeler AH, Goolkasian P, Gretz SS. Botulinum 
toxin A for the treatment of chronic neck pain. Pain 2001;94:255-60.

7. Li Q. Effect of forest bathing trips on human immune function. Environ Health Prev Med 2010;15:9-17.

8. Lee J, Park BJ, Tsunetsugu Y, Ohira T, Kagawa T, Miyazaki Y. Effect of forest bathing on physiological and psychological responses in young Japanese male subjects. Public Health 2011;125:93-100.

9. Sung J, Woo JM, Kim W, Lim SK, Chung EJ. The effect of cognitive behavior therapy-based "forest therapy" program on blood pressure, salivary cortisol level, and quality of life in elderly hypertensive patients. Clin Exp Hypertens 2012;34:1-7.

10. Park HS, Shin CS, Yeoun PS, Kim JY. A comparative study of the stress recovery effect of forest therapy. J Korean Inst For Recreat 2014;18:13-24.

11. Lee SI, Shin WS, Kwon YM, Kwon HG. The effect of woods aromatic substance. J Korean Inst For Recreat 2003;7:25-9.

12. Shin YK, Baik JH, Chae JH. Application of environmental therapy: focused on forest therapy and hydro- therapy. Korean J Str Res 2010;18:167-79.

13. Ylinen J. Physical exercises and functional rehabilitation for the management of chronic neck pain. Eura Medicophys 2007;43:119-32.

14. Douglass AB, Bope ET. Evaluation and treatment of posterior neck pain in family practice. J Am Board Fam Pract 2004;17 Suppl:S13-22.

15. Reips UD, Funke F. Interval-level measurement with visual analogue scales in Internet-based research: VAS Generator. Behav Res Methods 2008;40:699-704.

16. Vernon H, Mior S. The Neck Disability Index: a study of reliability and validity. J Manipulative Physiol Ther 1991;14:409-15.

17. Szende A, Oppe M, Devlin NJ. EQ-5D value sets: inventory, comparative review and user guide. Dordrecht: Springer; 2007.

18. Melzack R. The McGill Pain Questionnaire: major properties and scoring methods. Pain 1975;1:277-99.

19. Baldry P. Management of myofascial trigger point pain. Acupunct Med 2002;20:2-10. 